PAPER

\title{
Autosomal dominant cerebellar ataxia: SCA2 is the most frequent mutation in eastern India
}

\author{
K K Sinha, P F Worth, D K Jha, S Sinha, V J Stinton, M B Davis, N W Wood, M G Sweeney, \\ K P Bhatia
}

J Neurol Neurosurg Psychiatry 2004;75:448-452. doi: 10.1136/jnnp.2002.004895

See end of article for authors' affiliations

Correspondence to:

Dr K P Bhatia, Department of Clinical Neurology, Institute of Neurology,

Queen Square, London

WCIN 3BG, UK;

k.bhatia@ion.ucl.ac.uk
Objective: Spinocerebellar ataxia type 2 (SCA2) has been reported as the commonest dominant hereditary ataxia in India. However, India is an ethnically and religiously diverse population. Previous studies have not clearly indicated exact ethnic and religious origins, and must therefore be interpreted with caution. The purpose of this study was to determine the prevalence of different SCA mutations in a relatively homogeneous population from eastern India.

Methods: We identified 28 families with autosomal dominant cerebellar ataxia from eastern India. Each underwent full clinical evaluation and were analysed for the presence of SCA1, SCA2, SCA3, SCA6, SCA7, SCA8, SCA12, and SCA17 mutations. In addition, haplotype analysis was carried out in seven of the 16 families with SCA2.

Results: Seven patients from four (14\%) families were positive for an expansion in SCA1 and 26 patients from 16 (57\%) families were positive for an expansion in SCA2. No mutations were detected in the remaining eight families (29\%). Most of the SCA1 and SCA2 families were Hindu from the state of Bihar. Five out of 26 SCA2 patients in this study did not have slow saccades. In addition, four of seven SCA1 patients had slow saccades. We found an association between the SCA2 CAG repeat expansion and the 285 base pair (bp) allele of microsatellite marker D12S1672, and also data supportive of the association between the expansion and the 225 bp allele of D12S1333, which has been previously described.

Conclusions: We conclude that (1) although slow ocular saccades are highly suggestive of SCA2, that they are not universal, nor are they exclusive to this disorder and (2) SCA2 is likely to be the commonest dominant ataxia in eastern India, with further evidence for a founder effect.
W adia and Swami ${ }^{1}$ were the first to describe the early appearance of slow saccadic eye movements and peripheral neuropathy in what was then believed to be a unique form of autosomal dominant ataxia. Wadia suggested that this disorder, subsequently found to be spinocerebellar ataxia type 2 (SCA2), ${ }^{2}$ is the most frequently occurring form of hereditary ataxia, including Friedreich's ataxia, in western India. In a follow up study of six families from western India, Wadia et al have shown that the 26 members who all had slow horizontal saccadic eye movements in addition to ataxia were positive for an expansion at the SCA2 locus. ${ }^{3}$ The authors suggested that this clinical feature is strongly predictive of the SCA2 genotype. Haplotype analysis has subsequently shown the segregation of an identical core haplotype in these pedigrees as well as in those from Japan and England, ${ }^{4}$ suggesting that these SCA2 disease chromosomes are descended from a single ancestral chromosome.

Subsequent to the studies of Wadia et al the relative frequencies of the different CAG repeat expansions in various Indian populations have been studied by molecular analysis of DNA from patients from various parts of India for the different spinocerebellar ataxia (SCA) mutations. ${ }^{5-8}$ Most of these $^{5-7}$ have found SCA2 to be the commonest mutation. However, some studies do not clearly indicate the precise ethnic and/or geographical origin of the families. Hence these findings should be interpreted with this proviso in mind.

In order to determine the prevalence and origin of the different genotypes in eastern India we have identified 28 families with autosomal dominant cerebellar ataxia (ADCA) and analysed affected members for the presence of the different SCA mutations, and analysed the haplotypes of
ADCA families with the most frequently occurring mutation, that of SCA2. We have also investigated the accuracy of prediction of the genotype in a given patient, on the basis of the clinical features, in particular the presence of slowed ocular saccades and upper limb areflexia.

\section{METHODS AND MATERIALS}

\section{Clinical ascertainment and evaluation}

We identified 44 patients from 28 families. These patients underwent full neurological examination by three neurologists (KKS, DKJ, and SS) between October 1996 and January 1999. The geographical origin and religion of the 20 families subsequently found to have a SCA mutation is shown in table 1 . Most families were from the state of Bihar, and were Hindu. The mean age at onset, presenting symptoms, disease duration, and age at which patients became non-ambulant was recorded. In addition some patients underwent computed tomography (CT) brain scan, peripheral electromyography (EMG), and nerve conduction studies (NCS).

\section{Genetic analysis}

Although more than one affected family member was ascertained in the four SCA1 and 15/16 SCA2 families, DNA was available from only one individual in two SCAl and nine SCA2 families. DNA was extracted from peripheral blood lymphocytes using standard techniques. DNA was analysed

\footnotetext{
Abbreviations: $A D C A$, autosomal dominant cerebellar ataxia; $C T$, computed tomography; EMG, electromyography; NCS, nerve conduction studies; PCR, polymerase chain reaction; SCA, spinocerebellar ataxia
} 
Table 1 Religion and geographical origin of SCA1 and SCA2 families

\begin{tabular}{lll}
\hline & \multicolumn{2}{l}{ Number of families } \\
\cline { 2 - 3 } & SCA1 & SCA2 \\
\hline $\begin{array}{l}\text { Geographical origin* } \\
\text { Madhya Pradesh }\end{array}$ & 1 & 0 \\
$\quad$ Bihar & 3 & 15 \\
West Bengal & 0 & 1 \\
Religion & 3 & 14 \\
$\quad$ Hindu & 1 & 1 \\
Muslim & 0 & 1 \\
Tribal Christian & & \\
\hline
\end{tabular}

*In the three eastern Indian states.

SCA, spinocerebellar ataxia.

for SCA1, SCA2, SCA3, SCA6, SCA7, SCA8, SCA12, and SCA17 mutations according to established protocols. Polymerase chain reaction (PCR) products were checked on a $4 \%$ agarose mini gel before analysis on an ABI 377 DNA sequencer equipped with Genescan (ABI, Warrington, UK) software.

\section{Haplotype analysis}

Individuals from 11 of the 16 families were analysed. In four of these, DNA was available from only one family member. The remaining five families with DNA from only one affected were not analysed. Samples were analysed for four polymorphic microsatellite markers which map to chromosome $12 \mathrm{q}$, the relative order of which is as follows: D12S1328-(0.03 cM)D12S1332-(0.00 cM)-D12S1672-(0.00 cM)-D12S1333; the order and location of these markers is as in the Genetic Location Database (LDB). The SCA2 triplet repeat motif is located approx $20 \mathrm{~kb}$ distal and $200 \mathrm{~kb}$ proximal to D12S1672 and D12S1333, respectively. PCR amplification of the markers was carried out according to conditions described on the Genome Database (http://www.gdb.org). Fluorescent forward primers were used in the PCR reactions. PCR products were run on an ABI 377 automated DNA sequencer. Alleles were typed according to the CEPH reference sample 134702 and assigned numbers as used by Pang et al. ${ }^{4}$ Alleles determined which were not already allocated numbers were designated using the next consecutive number. Haplotypes were constructed assuming the least possible number of recombinations.

\section{Statistical analysis}

Correlation between age at onset and number of CAG repeats in SCAl and SCA2 was carried out using the Pearson correlation. Analysis of differences in clinical features between SCAl and SCA2 was carried out using the $\chi^{2}$ test.

\section{RESULTS}

\section{Comparative frequencies of different SCA mutations}

Of 28 families analysed, four (14\%) were positive for an expansion in SCAl and $16(57 \%)$ were positive for an expansion in SCA2. There was a clear family history of the disease in all families in whom a genetic diagnosis was made, except for one SCA2 positive patient, in whose family there was no history of neurological disease. No mutations were detected in the remaining eight ADCA type I families (29\%); in particular, no expanded SCA3, SCA6, SCA7, SCA8, SCA12, or SCA17 alleles were detected.

\section{Distribution of normal and pathological alleles}

Among SCAl patients, the range of repeat length of the pathological allele was 50-65 CAG repeats, while among SCA2 patients the range was $37-64$ repeats. In SCAl patients, the normal allele contained between 26 and 33 repeats, while in SCA2 patients the normal allele had 22 repeats in all cases. No individuals homozygous for an expanded SCAl or SCA2 allele were identified.

\section{Meiotic instability}

There were two paternal and five maternal SCAl transmissions. No SCAl parent-offspring pairs were available for analysis. In contrast, there were 13 paternal and 10 maternal SCA2 transmissions. In three SCA2 patients, the parent of origin could not be determined. In five cases of SCA2 transmission, the number of CAG repeats in the pathological allele could be determined; all five showed meiotic instability, and all were paternally transmitted. The mean change in repeat number was $+5.2(10.2)$ (range -3 to +19$)$ where "+" signifies an expansion during meiosis. The greatest meiotic instability was observed in a family where a daughter whose age at onset was 4 years inherited the disease from her father whose age at onset was 25 years. This paternally derived 45 repeat allele underwent expansion by 19 repeats.

One SCA2 patient with no family history of disease and whose age at onset was 15 years had a pathological allele of 58 CAG repeats. He had no affected siblings and his parents are both alive and said to be healthy although they have not been formally examined; DNA was not available.

\section{Clinical features of SCA 1 and SCA2 patients}

All 44 patients from 28 families had clinical features consistent with ADCA type I. The clinical features of the SCA1 and SCA2 families are summarised in table 2. Presenting clinical symptom was unsteadiness of gait in all SCAl patients but only 18/26 (69\%) SCA2 patients. Other presenting symptoms in SCA2 were tremor of head or hands in three $(12 \%)$, dysarthria in three $(12 \%)$, generalised weakness in two $(8 \%)$ and manual incoordination in one (4\%). The mean age at onset was similar in SCAl (23.9 (12.2) years) and SCA2 (24.4 (11.4) years) patients. There was a significant inverse correlation between age at onset and

Table 2 Clinical features of patients with expansions at the SCA1 and SCA2 loci

\begin{tabular}{lll} 
& \multicolumn{3}{l}{ SCA1 positive } & SCA2 positive \\
\hline Number of patients & 7 & 26 \\
Number of families & 4 & 16 \\
Age at onset; mean (SD) & $23.9(12.2)$ & $24.4(11.4)$ \\
(range) & $(18-51)$ & $(4-47)$ \\
Disease duration; mean (SD) & $6.0(5.2)(1-14)$ & $7.7(6.4)(1-27)$ \\
(range) & & \\
& Number of patients $(\%)$ \\
Dysarthria & $7(100)$ & $25(96)$ \\
Dysphagia & $2(29)$ & $2(8)$ \\
Gait ataxia & $7(100)$ & $25(96)$ \\
Upper limb ataxia & $7(100)$ & $26(100)$ \\
Lower limb ataxia & $7(100)$ & $26(100)$ \\
Ophthalmoplegia & $1(14)$ & $0(0)$ \\
Slow saccades & $4(57)$ & $21(81)$ \\
Nystagmus & $0(0)$ & $0(0)$ \\
Limitation of vertical gaze & $2(29)$ & $3(11)$ \\
Limitation of horizontal gaze & $2(29)$ & $2(8)$ \\
Eyelid retraction & $1(14)$ & $0(0)$ \\
Diplopia & $1(14)$ & $0(0)$ \\
Spasticity & $1(14)$ & $1(4)$ \\
Upper limb weakness & $1(14)$ & $3(11)$ \\
Lower limb weakness & $1(14)$ & $4(15)$ \\
Hyperreflexia & $3(43)$ & $4(15)$ \\
Hyporeflexia & $3(43)$ & $19(73)$ \\
Positive Babinski & $2(29)$ & $3(11)$ \\
Extrapyramidal signs & $0(0)$ & $1(4)$ \\
\hline SCA, spinocerebellar ataxia; SD, standard deviation. \\
\hline
\end{tabular}



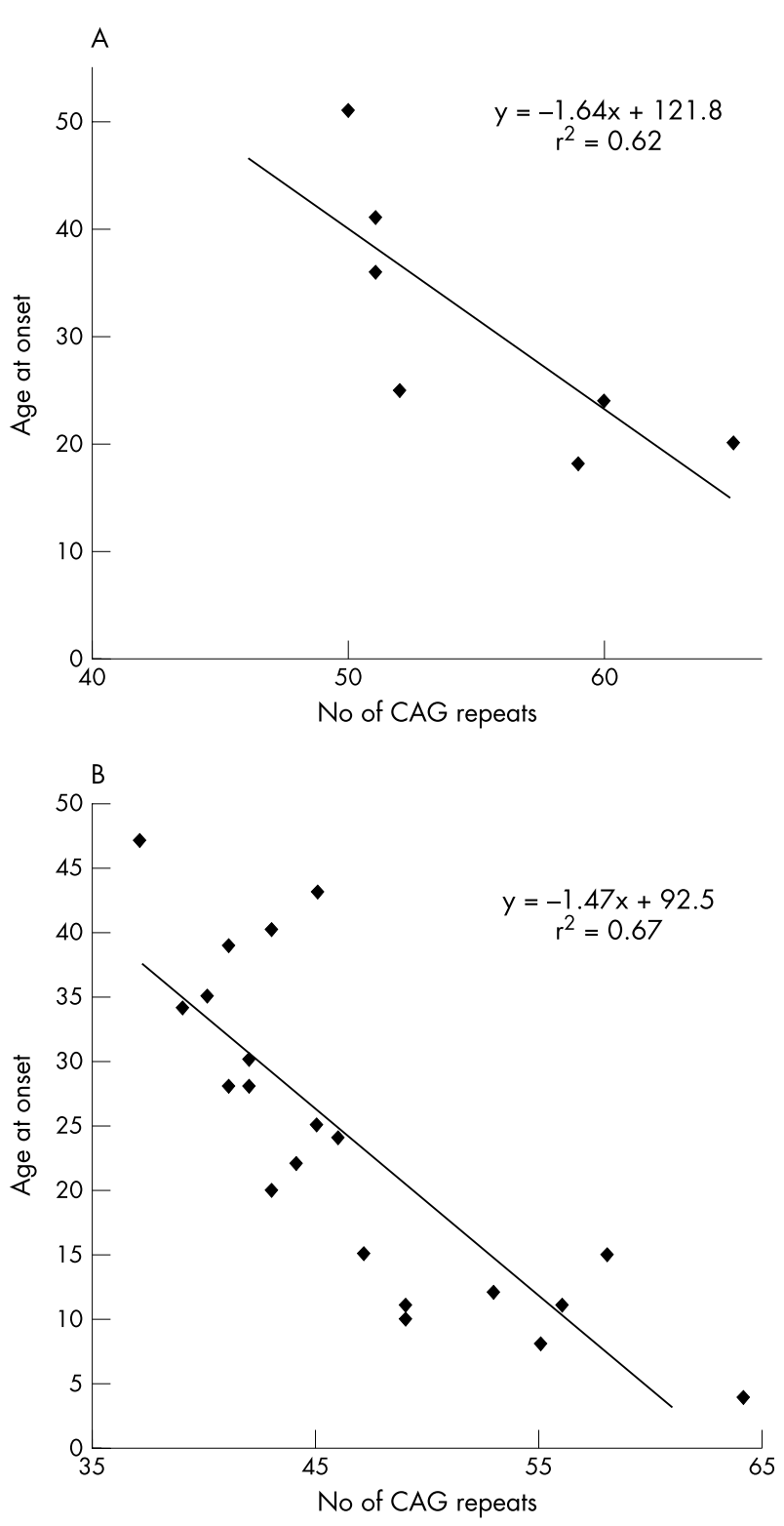

Figure 1 Plot of age at onset against number of $C A G$ repeats for $\mathrm{SCA} 1$ (A) and SCA2 (B). Statistically significant correlation is identified for both SCA1 and SCA2. The equation of each line is shown together with the $r^{2}$ value from the Pearson correlation.

number of CAG repeats in both SCAl $\left(\mathrm{r}^{2}=0.62, \mathrm{p}=0.036\right)$ and SCA2 patients $\left(\mathrm{r}^{2}=0.67, \mathrm{p}<0.0001\right)$ (fig 1 ).

Mean disease duration was well matched between the SCA1 and SCA2 patients, allowing comparison of clinical features. Although the number of SCAl patients is small, features which appeared to distinguish between the two groups included the presence of slow saccades and hyporeflexia; both features affected more SCA2 patients as compared to SCAl, although neither difference was statistically significant owing to the small SCAl sample size (slow saccades $\chi^{2}=0.64, \quad p=0.42 ; \quad$ hyporeflexia $\chi^{2}=0.71$, $\mathrm{p}=0.40$ ). Deep sensory disturbance was also much more common among SCA2 than SCAl patients.

\section{Investigations}

Two SCAl and seven SCA2 patients underwent EMG/NCS. All of these individuals had evidence of predominantly
Table 3 Haplotype analysis in seven unrelated SCA2 pedigrees with microsatellites which flank the SCA2 locus

\begin{tabular}{|c|c|c|c|c|}
\hline \multirow{2}{*}{$\begin{array}{l}\text { SCA2 } \\
\text { pedigrees }\end{array}$} & \multicolumn{4}{|c|}{ Allele marker } \\
\hline & D12S1328 & D12S1332 & D12S1672 & CAG repeat D12S1333 \\
\hline 1 & 3 & 3 & 4 & $2 / 3^{*}$ \\
\hline$\|$ & $4 / 2$ & 3 & $2 / 9$ & $2^{*}$ \\
\hline III & $3 / 7^{*}$ & $3 / 11^{*}$ & $8^{*}$ & $2^{*}$ \\
\hline IV & $3^{*}$ & $3^{*}$ & $8^{*}$ & $2 / 5^{*}$ \\
\hline V & $3 / 4^{*}$ & $3^{*}$ & $8 *$ & $2 / 5^{\star}$ \\
\hline $\mathrm{VI}$ & $3 / 7^{*}$ & $3^{*}$ & $8 / 9^{*}$ & $2 / 5^{*}$ \\
\hline VII & $4 / 8$ & $3 / 7$ & $2 / 9$ & $2 / 5^{*}$ \\
\hline
\end{tabular}

Seven pedigrees in which DNA from more than one individual was available (pedigrees I-VII) are shown. The remaining nine families with DNA from only one affected were not analysed. Alleles were assigned with reference to CEPH individual 134702. Where alleles were not previously described, the next available number was assigned. Where phase was not determinable, the genotype segregating with the disease is given.

*Indicates possible common disease associated haplotypes.

SCA2, spinocerebellar ataxia type 2 .

sensory axonal peripheral neuropathy, as previously described. In SCA2 patients, upper limb abnormalities were usually more marked than lower limb. Three SCAl and 13 SCA2 patients underwent CT brain scans. All had variable degrees of cerebellar and brainstem atrophy, and there were no discernible differences between the two groups.

\section{SCA2 haplotype analysis}

There were seven SCA2 pedigrees where the haplotype of more than one affected individual could be determined. The haplotypes of the SCA2 families are shown in table 3. It was not possible to determine phase for all markers in any of these pedigrees, although in two families, the allele segregating with the disease was identified for three of the four markers analysed. Although the data are too few for formal statistical analysis, allele 3 (202 bp) of D12S1332 segregates with the disease in at least five $(71 \%)$ and possibly all of the seven pedigrees. Allele 8 (285 bp) of marker D12S1672 segregates with the disease in at least three $(43 \%)$ but possibly four $(57 \%)$ out of seven SCA2 pedigrees. Allele 2 (225 bp) of marker D12S1333 is associated with the disease in at least two $(29 \%)$ and possibly all of these families. The core haplotype 3-8-2 (D12S1332-D12S1672-D12S1333) may be segregating with the disease in four families $(57 \%)$, although phase of the D12S1333 allele cannot be determined in three of these. A range of haplotypes is found among the SCA2 families where only one individual can be typed is observed, with no definite association between any one allele and the disease phenotype. Therefore, although an association in these SCA2 families between the 3-8-2 haplotype and the SCA2 CAG repeat appears possible, this cannot be shown conclusively.

\section{DISCUSSION}

In 1971 Wadia and Swami ${ }^{1}$ reported nine Indian families with a "new form of spinocerebellar degeneration", clinically characterised by early appearance of slow saccadic eye movements and peripheral neuropathy. Wadia et al have subsequently shown that six of these families from diverse geographical locations in western India have pathological expansions in SCA2. ${ }^{3}$ The authors showed that all 26 affected members had slow horizontal saccadic eye movements and argue that this feature is strongly predictive of the SCA2 genotype. Indeed, they suggest that a further 25 families with a similar phenotype characterised by slow saccades will also be shown to harbour an expansion at the SCA2 locus, though this remains unproven at present. None of the other SCA 
Table 4 A summary of the frequencies of different SCA mutations in previous studies in the Indian population

\begin{tabular}{|c|c|c|c|c|c|c|c|c|c|c|}
\hline & \multicolumn{3}{|c|}{$\begin{array}{l}\text { Geographic origin (no of } \\
\text { families) }\end{array}$} & \multicolumn{7}{|c|}{ No of families with each mutation (\%) } \\
\hline & North & East & NS & SCA1 & SCA2 & SCA3 & SCA6 & SCA7 & SCA12 & $U$ \\
\hline Saleem et al ${ }^{6}$ & 35 & 7 & - & 3 & 10 & 2 & 0 & 0 & - & 24 \\
\hline Basu et $a l^{5}$ & - & 26 & - & 5 (19.2) & 7 (26.9) & $3(11.5)$ & $1(3.8)$ & $0(0)$ & - & $10(38.5)$ \\
\hline Chakravarty et al ${ }^{8}$ & - & 14 & - & $2(14.3)$ & $4(28.6)$ & $5(35.7)$ & - & $0(0)$ & - & $3(21.4)$ \\
\hline Srivastava et $a l^{7}$ & - & - & 77 & $12(15.6)$ & $19(24.7)$ & $2(2.6)$ & 0 & $2(2.6)$ & $5(6.5)$ & $37(48)$ \\
\hline Sinha ef $a l^{9}$ & - & 28 & - & $4(14.3)$ & $16(57.1)$ & $0(0)$ & $0(0)$ & $0(0)$ & $0(0)$ & $8(28.6)$ \\
\hline
\end{tabular}

The current study is highlighted in bold. SCA2 is the commonest mutation found in all studies apart from that of Chakravarty et al ${ }^{8}$ where SCA3 was the most frequent.

*These authors studied a total of 42 families with any family history of ataxia, not exclusively ADCA. They found that members of three families had the Friedreich's mutation. They do not state the geographic origin of these families. Hence we have not expressed the percentages of each SCA as a percentage of the total, as this would be incomparable to the other studies, all of which looked at families with ADCA exclusively.

NS, not specified in the paper; SCA, spinocerebellar ataxia; U, undiagnosed.

genes has been analysed in these families, and therefore the relative prevalences in western India remains unknown.

Sinha et $a l^{9}$ were the first to suggest that SCA2 is the commonest hereditary ataxia in eastern India. Most of the subsequent studies have provided evidence that SCA2 may be the commonest ADCA in India as a whole, ${ }^{5-7}$ although one study found SCA3 to be slightly more frequent among 14 ethnic Bengali families. ${ }^{8}$ The results of these studies are summarised in table 4.

Our results lend weight to the existing evidence that SCA2 is the most frequent mutation in eastern India, at least among subjects from the state of Bihar, with 16 families $(57 \%)$ positive for an expansion in SCA2. Four families (14\%) were positive for an expansion in SCAl, but none of the other SCA genotypes was encountered. The proportion of families that remain undiagnosed was $29 \%$ (11 patients from eight ADCA type I families).

All of the published studies of the relative frequency of SCA mutations in India, including this study, have relatively small sample sizes and are not population based. The population of India is highly ethnically diverse, even within smaller geographical regions of the country, and this compounds the problem of interpretation. In addition, the causative mutation remains unidentified in a substantial number of patients in all these studies. Interestingly, Srivastava and colleagues in New Delhi have suggested that SCA12 may be more common among the Indian population than in other ethnic groups. ${ }^{7}$ Five families out of 77 (6.5\%) with ADCA were positive for an expansion in SCA12. However, SCA2 was the commonest mutation identified, with 19 families $(24.7 \%)$ affected. Again, $48 \%$ of families were negative for all mutations, and the ethnic origin of the families is not given.

The absence of SCA3 expansions in our series is particularly noteworthy. In three other studies of Indian ADCA, the frequency of SCA3 is relatively low; $5 \%,{ }^{6} 7 \%,{ }^{5}$ and $2.6 \%{ }^{7}$ This is unexpected, given India's colonial connection with Portugal, where SCA3 is common. However, the SCA3/MJD mutation was not transmitted by Portuguese sailors or travellers to black African and Jewish Yemenite SCA3 patients as previously hypothesised ${ }^{10-12}$ since the core haplotype observed in these populations has not been found in Portuguese patients. ${ }^{13}$ Notwithstanding these observations, SCA3 appears to be more common among ethnic Bengalis, where five out of 14 families $(36 \%)$ had an expansion in SCA3. ${ }^{8}$

Previous studies have highlighted the different prevalence of the various SCA mutations throughout the world. The reasons for this difference are unclear, but the variations in observed frequency probably relate to regional founder effects. Takano et $a l^{14}$ have shown that that the different prevalence of the dominantly inherited ataxias and dentatorubropallidoluysian atrophy (DRPLA) in separate Caucasian and Japanese populations was related to the frequency of the larger normal alleles of the respective gene. At least six recent studies ${ }^{4}{ }^{615-18}$ have analysed microsatellite markers around the SCA2 CAG repeat in different populations in order to detect evidence for linkage disequilibriumthat is, whether a common "core" haplotype exists in SCA2 families from diverse ethnic backgrounds. Two of these found no evidence for a founder effect among the families analysed. ${ }^{15}{ }^{16}$ Among 26 SCA2 families from Europe, North Africa, and the Caribbean described by Didierjean et $a l^{18}$ evidence for several different founder mutations was determined. However, two French, one Serbian, and up to four German families shared one of the various identified haplotypes. Similarly, two further French and three Austrian families shared another haplotype.

There have been two previous studies of SCA2 in India where the background haplotypes have been analysed. Both of these studies have identified a strong association between SCA2 disease chromosomes and the $225 \mathrm{bp}$ allele of D12S1333, not only in Indians, but also in one Cuban, one English, and three Japanese families. In addition, the first of these $^{4}$ studies showed apparent linkage disequilibrium between expanded SCA2 mutations and the 283 bp allele of D12S1672 in 12 families from different countries. However, the $285 \mathrm{bp}$ allele of D12S1672 was found to be in phase with the SCA2 expansion in at least three of their seven Indian families from western India. In contrast, the second study ${ }^{6}$ reported association of the $281 \mathrm{bp}$ allele of D12S1672 with the expanded SCA2 CAG repeat, although the 283 bp allele was found on the disease chromosome in two families.

Our data suggest that the haplotype 3-8-2 (D12S1332D12S1672-D12S1333) may be in linkage disequilibrium with the pathological SCA2 expansion in up to four Indian families from eastern India. Our data are supportive of the finding by Pang et al that allele $8(285 \mathrm{bp})$ of D12S1672 is associated with the SCA2 expansion in Indian patients; it was found in at least three (43\%) and possibly four of our families and is very uncommon among Indian controls (only one out of 72 control chromosomes). ${ }^{4}$ Interestingly, Saleem et $a l^{6}$ report linkage disequilibrium between the $281 \mathrm{bp}$ allele of D12S1672 and the SCA2 CAG repeat, although this allele is only 4 bp shorter than allele 8 . Allele 3 of D12S1332 was also common among our SCA2 pedigrees (71\%) but is also relatively common among Indian control chromosomes (33\%). We are confident that incorrect sizing of alleles does not account for these findings since a CEPH control individual was used in all three studies.

In summary, although the 225 bp allele of D12S1333 was strongly associated with the SCA2 phenotype two previous studies, our data are insufficient to confirm that this allele, although represented in all seven pedigrees, was associated 
with the SCA2 expansion, given the indeterminate phase in five of the seven families analysed. Interestingly, this allele is relatively rare among European, North African, and French west Indian SCA2 families, ${ }^{18}$ and is present in between $4 \%$ and $11 \%$ of Indian, Cuban, and Japanese controls. However, our results are consistent with the other two studies with respect to this allele. In addition, the apparent independent association of alleles $281 \mathrm{bp}, 283 \mathrm{bp}$, and $285 \mathrm{bp}$ of D12S1672 with the SCA2 CAG repeat reported in this study and the previous two studies, could have resulted from successive microsatellite slippage events or separate ancestral mutational events.

The comparison of the clinical features of SCA1 and SCA2 patients confirm that no single clinical sign can reliably distinguish between the different SCAs, although among this population, $81 \%$ of SCA2 and $57 \%$ of SCA1 patients had slow saccades. Hyporeflexia was also more common among SCA2 patients $(73 \%)$ than SCAl $(43 \%)$, although this difference was not statistically significant.

In conclusion, our data show that SCA2 is the most frequently occurring SCA in eastern India and reaffirms SCA2 as the predominant SCA in India overall. Our haplotype data are supportive of a common ancestral chromosome among the Indian population as found by Pang et $a l^{4}$ and Saleem et $a l^{6}$ Although we confirm that slow saccades and hyporeflexia are suggestive of SCA2, they are not unique to this phenotype, and genetic diagnosis is always to be recommended.

\section{ACKNOWLEDGEMENTS}

We would like to thank the families for their participation in this study.

\section{Authors' affiliations}

P F Worth, V J Stinton, M B Davis, N W Wood, M G Sweeney, K P Bhatia, Department of Clinical Neurology, Institute of Neurology, Queen Square, London WCIN 3BG, UK

K K Sinha, D K Jha, S Sinha, Department of Neurology, Advanced Diagnostic Centre, Booty Road, Bariatu, Ranchi-834 009, Bihar, India

\section{REFERENCES}

1 Wadia NH, Swami RK. A new form of heredo-familial spinocerebellar degeneration with slow eye movements (nine families). Brain 1971;94:359-74.
2 Pulst SM, Nechiporuk A, Nechiporuk T, et al. Moderate expansion of a normally biallelic trinucleotide repeat in spinocerebellar ataxia type 2. Nat Genet 1996;14:269-76

3 Wadia N, Pang J, Desai J, et al. A clinicogenetic analysis of six Indian spinocerebellar ataxia (SCA2) pedigrees. The significance of slow saccades in diagnosis. Brain 1998;121:2341-55.

4 Pang J, Allotey R, Wadia N, et al. A common disease haplotype segregating in spinocerebellar ataxia 2 (SCA2) pedigrees of diverse ethnic origin. Eur J Hum Genet 1999;7:841-5.

5 Basu P, Chattopadhyay B, Gangopadhaya PK, et al. Analysis of CAG repeats in SCA1, SCA2, SCA3, SCA6, SCA7 and DRPLA loci in spinocerebellar ataxia patients and distribution of CAG repeats at the SCA1, SCA2 and SCA6 loci in nine ethnic populations of eastern India. Hum Genet 2000; 106:597-604.

6 Saleem Q, Choudhry S, Mukerii M, et al. Molecular analysis of autosomal dominant hereditary ataxias in the Indian population: high frequency of SCA2 and evidence for a common founder mutation. Hum Genet 2000; 106:179-87.

7 Srivastava AK, Choudhry S, Gopinath MS, et al. Molecular and clinical correlation in five Indian families with spinocerebellar ataxia 12. Ann Neurol 2001;50:796-800.

8 Chakravarty A, Mukherjee SC. Autosomal dominant cerebellar ataxias in ethnic Bengalees in West Bengal-an Eastern Indian state. Acta Neurol Scand 2002;105:202-8.

9 Sinha K, Bhatia K, Sweeney M, et al. The frequency and phenotype-genotype correlation in 18 families with autosomal dominant cerebellar ataxia type I (ADCA-I) from eastern India. Neurology 1998;50:A170.

10 Rosenberg RN. Joseph disease: an autosomal dominant motor system degeneration. Adv Neurol 1984;41:179-93.

11 Rosenberg RN. Machado-Joseph disease: an autosomal dominant motor system degeneration. Mov Disord 1992;7:193-203.

12 Sequeiros J, Coutinho P. Epidemiology and clinical aspects of MachadoJoseph disease. Adv Neurol 1993;61:139-53.

13 Stevanin G, Cancel G, Didierjean O, et al. Linkage disequilibrium at the Machado-Joseph disease/spinal cerebellar ataxia 3 locus: evidence for a common founder effect in French and Portuguese-Brazilian families as well as a second ancestral Portuguese-Azorean mutation. Am J Hum Genet 1995; $57: 1247-50$

14 Takano H, Cancel G, Ikeuchi T, et al. Close associations between prevalences of dominantly inherited spinocerebellar ataxias with CAG-repeat expansions and frequencies of large normal CAG alleles in Japanese and Caucasian populations. Am J Hum Genet 1998;63:1060-6.

15 Geschwind $\mathrm{DH}$, Perlman S, Figueroa $\mathrm{CP}$, et al. The prevalence and wide clinical spectrum of the spinocerebellar ataxia type 2 trinucleotide repeat in patients with autosomal dominant cerebellar ataxia. Am J Hum Genet 1997;60:842-50.

16 Giunti P, Sabbadini G, Sweeney MG, et al. The role of the SCA2 trinucleotide repeat expansion in 89 autosomal dominant cerebella ataxia families. Frequency, clinical and genetic correlates. Brain 1998; 121:459-67.

17 Mizushima K, Watanabe M, Abe K, et al. Analysis of spinocerebellar ataxia type 2 in Gunma prefecture in Japan: CAG trinucleotide expansion and clinical characteristics. J Neurol Sci 1998;156:180-5.

18 Didierjean O, Cancel G, Stevanin G, et al. Linkage disequilibrium at the SCA2 locus. J Med Genet 1999;36:415-7. 